\title{
Considering the major mental disorders as clinical expressions of periodic pathological oscillations of the overall operating mode of brain function the article itself Nichoalas Pediaditakis
}

\author{
Address: Department of Psychiatric Medicine, East Carolina University School of Medicine, Greenville, NC, USA \\ from International Society on Brain and Behaviour: 3rd International Congress on Brain and Behaviour \\ Thessaloniki, Greece. 28 November - 2 December 2007 \\ Published: 17 April 2008 \\ Annals of General Psychiatry 2008, 7(Suppl I):SI02 doi:I0.I I86/I744-859X-7-SI-SI 02
}

This abstract is available from: http://www.annals-general-psychiatry.com/content/7/SI/SI02

(C) 2008 Pediaditakis; licensee BioMed Central Ltd.

\section{Background}

Studying shared clinical phenomena in major mental disorder. [1]

\section{Materials and methods}

Evidence from 50 years of evaluating shared characteristics and overlaps in clinical expressions and pharmacological responses [2] will be used to consider their collective significance in major mental disorders.

\section{Results}

1. These disorders have a common initial neurodevelopmental origin.

2. They occur probabilistically on some of "at-risk" individuals whose pre-existing underlying structural variance (expressed as temperament) confers vulnerability for such occurrences.

3. They can be considered as clinical expressions of pathologically ordered phase of the overall operating mode of brain function, expressed in the characteristic symptoms for each, made up of recurrent antithetical substitutes i.e., mania /depression, apathy/ explosively, obsessiveness/ slovenliness either-or thinking etc..[3]

4. This mode based on a particular for our brain algorithm (as an emergent quality of complexity) normally ensures, within limits, the synchrony, coordination, amalgamated subtlety and robust flexibility during the expression of each of the higher faculties. Specifically, mood modulation, coordination of feeling and thoughts, the rules of thinking, sequencing/ scheduling and appropriate responses to the external world. [4]

\section{Conclusions}

Such consideration opens opportunities for novel, therapies such as an input of specific, electric signal or magnetic field (versus the crude effect of ECT) or by noise cancellation techniques, restoring and maintaining the normal function of the operating modeIt also challenges us into re-thinking the current nosological, procrustean, flawed classification which prevents us from discerning the collective significance of these phenomena.

\section{Acknowledgements}

My gratitude and appreciation to Nicholas Stratas, MD, DLFAPA, David Servan-Schreiber, MD, PhD, Robert Webbie, MD, PhD, Peter Pediaditakis, $\mathrm{PhD}$, and John Rather, $\mathrm{PhD}$ for helping and advising me as to how to best express my concepts in the paper and my daughter, Nicole for endlessly editing and correcting my article.

\section{References}

I. Pediaditakis N: Considering the major mental disorders as clinical expressions of periodic pathological oscillations of the overall operating mode of brain function. Med Hypotheses 2006, 67:395-400.

2. Pediaditakis N: Shared characteristics in the clinical expression and pharmacological responses of mental disorders and their possible collective significance. Med Hypotheses 1998, 50:347-352.

3. Pediaditakis N: Deterministic nonlinear chaos in brain function and borderline psychopathological phenomena. Med Hypotheses 1992, 39:67-72

4. Pediaditakis N: The occurrence of schizophrenia in monozygotic twins and fractal, dentritic development. Arch Gen Psychiat 1996, 53:85. 